DESY 04-130

July 22, 2004

\title{
A Comment on the Cosmological Constant Problem in Spontaneously Broken Supergravity
}

\author{
A. Hebecker \\ Deutsches Elektronen-Synchrotron, Notkestraße 85, D-22603 Hamburg, Germany \\ ( arthur.hebecker@desy.de)
}

\begin{abstract}
In spontaneously broken supergravity with non-flat potential the vanishing of the cosmological constant is usually associated with a non-trivial balancing of two opposite-sign contributions. We make the simple observation that, in an appropriately defined expansion of the superfield action in inverse powers of $M_{P}$, this tuning corresponds to the absence of two specific operators. It is then tempting to speculate what kind of nonstandard symmetry or structural principle might underlie the observed extreme smallness of the corresponding coefficients in the real world. Independently of such speculations, the suggested expansion appears to be a particularly simple and convenient starting point for the effective field theory analysis of spontaneously broken supergravity models.
\end{abstract}


Supersymmetry or, more specifically, supergravity has so far not been able to offer a solution to the fundamental problem of the smallness of the cosmological constant. In fact, when compared with a simple non-SUSY model of gravity and a scalar field $\phi$, the situation might even look worse in the following sense. Expanding the non-SUSY lagrangian in inverse powers of the Planck mass $M_{P}$ (and in powers of $\phi$, as far as the scalar field part is concerned), one finds that the cosmological constant problem is characterized by the anomalous smallness of the coefficient of $\operatorname{det}\left(g_{\mu \nu}\right)$. However, even in the simplest version of spontaneously broken supergravity, involving just one chiral matter superfield, one apparently has to balance a positive and a negative contribution to the potential (cf. Eq. (4) below). At the minimum of the potential, these two contributions, each of which is derived in a non-trivial way from the fundamental action, have to cancel with very high precision. The only known cases where this cancellation is achieved for structural reasons, the so-called no-scale models, suffer from the presence of an exactly massless scalar field.

In this short note we point out that it is possible to define an expansion of the supergravity action in inverse powers of $M_{P}$ in such a way that the requirement of a SUSY-breaking vacuum with a vanishing cosmological constant amounts simply to the absence of two specific operators. In short, using a Kähler-Weyl frame where the superpotential is constant and expanding the function $\exp K(\Phi, \bar{\Phi})$ in powers of the chiral superfield $\Phi$, one has to forbid the terms $\Phi \bar{\Phi}$ and $\left(\Phi^{2} \bar{\Phi}+\right.$ h.c. $)$. As expected, there is no obvious symmetry principle enforcing this form, but a certain suggestive structure excluding the two dangerous operators will be identified below. On the one hand, these observations are extremely simple and their value might be merely pedagogical. On the other hand, one might hope that a deeper structural understanding of supergravity and its UV completion will emerge and justify the specific constraints mentioned above and to be discussed in more detail in the following.

To be more specific, we start with the superfield action for a supergravity model [1] with one chiral superfield $\Phi$ as it emerges from the curved superspace approach (see e.g. $[2,3])$. We follow the formulation of [4], which is very close to the more condensed treatment of [5] (see also [6]), and write the action as

$$
S=\int d^{4} x d^{2} \theta d^{2} \bar{\theta} E\left\{\Omega(\Phi, \bar{\Phi})+\left(\frac{1}{R} W(\Phi)+\text { h.c. }\right)\right\} .
$$

Here $E$ is the supervierbein determinant, $R$ is the superspace curvature, $W$ is the superpotential and $\Omega$, called the 'superspace kinetic energy' in [5], is related to the Kähler potential by $K=-3 \ln \left(-\Omega / 3 M^{2}\right)$ with the reduced Planck mass $M=M_{P} / \sqrt{8 \pi}$. The field $\Phi$ is covariantly chiral, $\overline{\mathcal{D}}_{\dot{\alpha}} \Phi=0$, and the same is true for $R$.

In flat space and setting all fermionic fields to zero, the above action takes the form

$$
S=\int d^{4} x d^{2} \theta d^{2} \bar{\theta} \varphi \bar{\varphi} \Omega(\Phi, \bar{\Phi})+\left(\int d^{2} \theta \varphi^{3} W(\Phi)+\text { h.c. }\right)+\cdots
$$

where $\Phi$ is now a flat-space chiral superfield. This simple form is made possible by introducing the chiral compensator [3-9] which, again in flat space and without fermions, is given (in 'gravitational superfield gauge' [4]) by $\varphi=1+\theta^{2} F_{\varphi}$. The ellipses in Eq. (2) stand 
for extra kinetic terms for the scalar component of $\Phi$ which originate from integrating out the auxiliary vector field and have no simple flat-superspace representation.

It is now easy to eliminate $F_{\Phi}$ and $F_{\varphi}$ and to obtain the scalar potential. Before doing so, we make the additional assumption that the superpotential is constant, $W(\Phi)=$ $c_{W} M^{3}$. At the moment, this can be done without loss of generality since, given that $W$ is non-zero in the vacuum, it can always be made constant by performing an appropriate Kähler-Weyl transformation. However, later on we will have to impose constraints on $\Omega$ and this simple basis choice will be promoted to a true constraint.

Thus, for $W(\Phi)=c_{W} M^{3}$, the scalar potential derived from Eq. (2) reads

$$
V_{\text {non-can. }}(\Phi, \bar{\Phi})=\frac{9\left|c_{W}\right|^{2} M^{6} \Omega_{\Phi \bar{\Phi}}}{\Omega_{\Phi \bar{\Phi}} \Omega-\left|\Omega_{\Phi}\right|^{2}},
$$

where, as is commonly done, we use the same symbol for the superfield $\Phi$ and its scalar component. The index 'non-can.' serves as a reminder that metric and scalar field are not canonically normalized. In particular, the Ricci-scalar enters the action with the coefficient $\Omega / 6$. The indices of $\Omega$ symbolize partial derivatives.

Of course, one could have found an equivalent result by starting from the well-known formula (valid for canonical field normalization)

$$
V(\Phi, \bar{\Phi})=\frac{1}{M^{2}} \exp (K)\left(\left(K_{\Phi \bar{\Phi}}\right)^{-1}\left|W_{\Phi}+K_{\Phi} W\right|^{2}-3|W|^{2}\right)
$$

assuming constant $W$, and expressing $K$ through $\Omega$. The utility of such a formulation has been recognized and exploited for a detailed analysis of positivity properties of the potential in [10] (see also [11]). We note that $V=\left(3 M^{2} / \Omega\right)^{2} V_{\text {non-can. }}$.

The crucial point now comes with the assumption that at $\Phi=0$ (which, as will be argued later, may be somehow distinguished at a fundamental level) the potential and its first derivatives vanish. In other words, the point $\Phi=0$ is a SUSY-breaking vacuum with zero cosmological constant. We want to analyse this assumption in the spirit of lowenergy effective field theory, i.e., expanding the action of Eq. (11) (and thus the function $\Omega)$ in powers of $\Phi / M$ :

$$
\begin{aligned}
\Omega(\Phi, \bar{\Phi})= & -3 M^{2}+\left(c_{\Phi} \Phi M+\text { h.c. }\right)+c_{\Phi \bar{\Phi}} \Phi \bar{\Phi}+\left(c_{\Phi^{2}} \Phi^{2}+\text { h.c. }\right) \\
& +\left(c_{\Phi^{2} \bar{\Phi}} \frac{\Phi^{2} \bar{\Phi}}{M}+\text { h.c. }\right)+\left(c_{\Phi^{3}} \frac{\Phi^{3}}{M}+\text { h.c. }\right)+\cdots
\end{aligned}
$$

It can now be easily seen from Eq. (3) that the above two assumptions simply mean that the coefficients $c_{\Phi \bar{\Phi}}$ and $c_{\Phi^{2} \bar{\Phi}}$ vanish. The constant term in Eq. (5) is used to define the Planck scale. The linear term is crucial for SUSY breaking and would, if the series were to end there, define a no-scale model [12]. (This would, of course, also be true if the series were to continue with only holomorphic and antiholomorphic terms.) The term $\Phi \bar{\Phi}$ must be zero to have vanishing vacuum energy and, given that this is the case, it is now only 
the term $\sim \Phi^{2} \bar{\Phi}$ which could produce a linear term in the scalar potential. Thus, it has to be forbidden as well, giving the scalar potential

$$
V(\Phi, \bar{\Phi})=-\frac{9\left|c_{W}\right|^{2} M^{2}}{\left|c_{\Phi}\right|^{2}}\left\{4 c_{\Phi^{2} \bar{\Phi}^{2}} \Phi \bar{\Phi}+\left(3 c_{\Phi^{3} \bar{\Phi}} \Phi^{2}+\text { h.c. }\right)+\mathcal{O}\left(|\Phi|^{3} / M\right)\right\} .
$$

This defines the scalar masses in terms of $c_{W}, c_{\Phi}$ and the coefficients $c_{\Phi^{2} \bar{\Phi}^{2}}, c_{\Phi^{3} \bar{\Phi}}$, which we assume to have values making the extremum at $\Phi=0$ stable. The gravitino mass is given by $\left|c_{W}\right| M$.

In summary, we have assumed that the action of Eq. (1), with $W$ being constant, gives rise to a flat, SUSY-breaking vacuum at $\Phi=0$. Expanding around this point in inverse powers $M$ (which is defined by the coefficient of the $\Phi$-independent term of $\Omega$ ), we have found that two of the coefficients have to vanish for consistency. Then we have identified gravitino and scalar masses in terms of the remaining coefficients. It should also be noted that, when deriving Eq. (3) in this setting, $F_{\varphi}$ is found to be zero in the vacuum.

We conclude with some comments concerning possible structural reasons for the absence of the two offending terms and on the generalization to more than one chiral matter superfield. It is easy to see that a 'superspace kinetic energy' of the form

$$
\Omega(\Phi, \bar{\Phi})=f(\Phi)+f(\bar{\Phi})+F\left(\Phi^{2}, \bar{\Phi}^{2}\right)
$$

automatically has vanishing $c_{\Phi \bar{\Phi}}$ and $c_{\Phi^{2} \bar{\Phi}}$. (Our notation implies that $f$ and $F$ are defined as power series of their respective arguments.) One way to look at this is to view $F$ as a very specific correction to a no-scale model, ensuring that the flatness is lifted while the zero vacuum energy is preserved. Another possible attitude is to consider $f$ as a correction to a model defined by the completely generic 'kinetic function' $F(\Psi, \bar{\Psi})$. This correction would have to be introduced in a very peculiar way, namely by adding the no-scale type function $f(\Phi)+f(\bar{\Phi})$ after the reparameterization $\Psi \rightarrow \Phi^{2}$. At the moment, neither of these possibilities may appear particularly convincing or easy to justify at the quantum level. However, we recall that a similar criticism may be applied, for example, to the ansatz of writing both superpotential and 'kinetic function' as the sum of hidden and visible sector contributions [13]. Yet, this turns out to be natural if the two sectors are separated in higher dimensions. In this spirit, one may hope that the ansatz of Eq. (7) or some similar ansatz justifying $c_{\Phi \bar{\Phi}}=c_{\Phi^{2} \bar{\Phi}}=0$ will eventually find a deeper understanding.

An obvious way to generalize the above discussion to more superfields is by adding a set of chiral fields $Q=\left\{Q^{i}\right\}$ which transform under some global, continuous, unitary symmetry. It is easy to see that, making all coefficients $c$ in Eq. (5) and $c_{W}$ arbitrary functions of $Q$ (respecting, of course, the symmetry) will not affect the existence of a SUSY-breaking stationary point at $\Phi=0$ and $Q=0$ with vanishing cosmological constant. The reason is the enhanced symmetry at this point, which makes it impossible to have linear terms driving either $Q$ or $F_{Q}$ to non-zero values. Of course, to make this point a minimum rather than just an extremum imposes further conditions on the coefficients in the lagrangian which, however, do not involve any fine-tuning. 
A more interesting generalization is to include further uncharged fields, so that all fields in the set $\left\{\Phi^{i}\right\}$ can contribute linear terms to $\Omega$ and mix with each other. Following the one-field case discussed above, we expand $\Omega$ around $\Phi^{i}=0$ (for all $i$ ) and require the presence of a linear contribution. As has been shown in [10] (cf. also [14]), the scalar potential is proportional to $\operatorname{det}\left[\Omega_{i \bar{j}}(\Phi, \bar{\Phi})\right]$. (Here the indices indicate derivatives with respect to $\Phi^{i}$ and $\bar{\Phi}^{j}$.) The vanishing of the cosmological constant at $\Phi^{i}=0$ corresponds to $\operatorname{det}\left[\Omega_{i \bar{j}}(0,0)\right]=0$, which is the generalization of the above condition $c_{\Phi \bar{\Phi}}=0$ to the multi-field case. The vanishing determinant of $\Omega_{i \bar{j}}(0,0)$ implies the existence of a vector $v^{\bar{i}}$ annihilated by this hermitian matrix, and we can choose a basis where only the first component of this vector is different from zero. In this basis, one has $\Omega_{1 \bar{i}}(0)=\Omega_{i \overline{1}}(0)=0$. This implies that the first derivative of $\operatorname{det}\left[\Omega_{i \bar{j}}\right]$ at the point $\Phi^{i}=0$ can only be non-zero if the first derivative of the element $\Omega_{1 \overline{1}}$ is non-zero at this point. Thus, given the above basis choice, the stationarity condition (the previous condition $c_{\Phi^{2} \bar{\Phi}}=0$ ) generalizes to $\Omega_{1 \overline{1} i}(0,0)=0$ in the multi-field case. This condition can also be given in the basis independent form $v^{i} \Omega_{i \bar{i} j}(0,0) v^{\bar{i}}=0$. Thus, it appears that a nonlinear relation between different coefficients in the expansion of $\Omega(\Phi, \bar{\Phi})$ has arisen. The simple form of the constraints found in the one-field case does not emerge unless one distinguishes a specific field which, as an a-priori feature, does not participate in any non-holomorphic secondorder terms in $\Omega$.

Acknowledgements: I would like to thank W. Buchmüller, S. Ferrara, and R. Sundrum for useful comments and suggestions. I am also grateful to B. Wonsak for many discussions during the preparation of his Diploma thesis [15].

\section{References}

[1] D. Z. Freedman, P. van Nieuwenhuizen and S. Ferrara, "Progress Toward A Theory Of Supergravity," Phys. Rev. D 13 (1976) 3214;

S. Deser and B. Zumino, "Consistent Supergravity," Phys. Lett. B 62 (1976) 335;

E. Cremmer, B. Julia, J. Scherk, P. van Nieuwenhuizen, S. Ferrara and L. Girardello, "Superhiggs Effect In Supergravity With General Scalar Interactions," Phys. Lett. B 79 (1978) 231; "Spontaneous Symmetry Breaking and Higgs Effect In Supergravity Without Cosmological Constant," Nucl. Phys. B 147 (1979) 105.

[2] J. Wess and B. Zumino, "Superspace Formulation Of Supergravity," Phys. Lett. B 66 (1977) 361; "Superfield Lagrangian For Supergravity," Phys. Lett. B 74 (1978) $51 ;$

V. Ogievetsky and E. Sokatchev, "On Vector Superfield Generated By Supercurrent," Nucl. Phys. B 124 (1977) 309; "Structure Of Supergravity Group," Phys. Lett. 79B (1978) 222.

[3] W. Siegel and S. J. J. Gates, "Superfield Supergravity," Nucl. Phys. B 147 (1979) 77. 
[4] I. L. Buchbinder and S. M. Kuzenko, Ideas and Methods of Supersymmetry and Supergravity, Inst. of Physics Publishing, Bristol, 1998.

[5] J. Wess and J. Bagger, Supersymmetry and Supergravity, Princeton Univ. Press, 1983.

[6] S. J. Gates, M. T. Grisaru, M. Rocek and W. Siegel, "Superspace, Or One Thousand And One Lessons In Supersymmetry," Front. Phys. 58 (1983) 1 arXiv:hep-th/0108200.

[7] E. Cremmer, S. Ferrara, L. Girardello and A. Van Proeyen, "Yang-Mills Theories With Local Supersymmetry: Lagrangian, Transformation Laws And Superhiggs Effect," Nucl. Phys. B 212 (1983) 413.

[8] T. Kugo and S. Uehara, "Improved Superconformal Gauge Conditions In The N=1 Supergravity Yang-Mills Matter System," Nucl. Phys. B 222 (1983) 125; "Conformal And Poincare Tensor Calculi In N=1 Supergravity," Nucl. Phys. B 226 (1983) 49.

[9] V. Kaplunovsky and J. Louis, "Field dependent gauge couplings in locally supersymmetric effective quantum field theories," Nucl. Phys. B 422 (1994) 57 arXiv:hep-th/9402005.

[10] R. Barbieri, E. Cremmer and S. Ferrara, "Flat And Positive Potentials In N=1 Supergravity," Phys. Lett. B 163 (1985) 143.

[11] L. Andrianopoli, R. D'Auria, S. Ferrara and M. A. Lledo, "Gauged extended supergravity without cosmological constant: No-scale structure and supersymmetry breaking," Mod. Phys. Lett. A 18 (2003) 1001 arXiv:hep-th/0212141.

[12] E. Cremmer, S. Ferrara, C. Kounnas and D. V. Nanopoulos, "Naturally Vanishing Cosmological Constant In N=1 Supergravity," Phys. Lett. B 133 (1983) 61;

J. R. Ellis, A. B. Lahanas, D. V. Nanopoulos and K. Tamvakis, "No - Scale Supersymmetric Standard Model," Phys. Lett. B 134 (1984) 429;

N. Dragon, M. G. Schmidt and U. Ellwanger, "Sliding Scales In Minimal Supergravity," Phys. Lett. B 145 (1984) 192; "Supersymmetry And Supergravity," Prog. Part. Nucl. Phys. 18 (1987) 1;

A. B. Lahanas and D. V. Nanopoulos, "The Road To No Scale Supergravity," Phys. Rept. 145 (1987) 1.

[13] L. Randall and R. Sundrum, "Out of this world supersymmetry breaking," Nucl. Phys. B 557 (1999) 79 arXiv:hep-th/9810155;

M. A. Luty and R. Sundrum, "Radius stabilization and anomaly-mediated supersymmetry breaking," Phys. Rev. D 62 (2000) 035008 arXiv:hep-th/9910202.

[14] S. P. Li, R. Peschanski and C. A. Savoy, "Mass Degeneracy And The Superhiggs Mechanism," Nucl. Phys. B 289 (1987) 206.

[15] B. S. Wonsak, "Superfield Supergravity and an Almost No-Scale Scenario," Diploma thesis, University of Hamburg, 2004, DESY-THESIS-2004-010. 